\title{
Foreign Material Causing Red Discoloration of the Lung: Energy Dispersive X- Ray Analysis
}

Suman Goel, M.D., M.P.H., LeRoy Riddick, M.D., and Judy King, M.D., Ph.D.

Department of Pathology, University of South Alabama, 2451 Fillingim Street, Mobile, Alabama 36617

The lungs filter 500 liters of air per hour, amounting to approximately 250 million liters over the lifetime of an individual [1]. This surrounding unfiltered air contains particles, gases and infectious agents. The inhaled substances, dependent on the size, shape, concentration, duration of exposure, and chemical nature of the particles, must either deposit on the mucosal surface or penetrate the epithelium to affect the lung.

Identification and quantitative analysis of the particulate burden may require an interdisciplinary approach involving patients, epidemiologists, clinicians, pathologists, toxicologists, and microscopists. Different techniques for mineral analysis include conventional bright-field light microscopy, phase-contrast microscopy, polarized microscopy and electron microscopy. However, only the electron microscope can precisely locate and determine the chemical composition of particles when coupled with electron diffraction or x-ray analysis. We report the analysis of an archived autopsy case with diffuse red discoloration of the lung (Fig. 1).

MATERIALS AND METHODS: Representative portions of the lung were routinely fixed in $10 \%$ neutral buffered formalin and embedded in paraffin. The foreign brown-black material was identified on hematoxylin \& eosin (H \& E) stained sections (Fig. 2). For scanning electron microscopy (SEM) and energy dispersive x-ray analysis (EDXA), 8 micron thick sections were cut from the paraffin block and either placed on a Thermanox (Fisher Scientific, Norcross, GA) coverslip or directly on the carbon stub. Coverslips with tissue were attached to stubs using carbon double-stick tabs. Specimens were coated with carbon, and examined in a Philips XL 20 SEM (FEI, Hillsboro, OR) with EDXA (EDAX, Mahwah, NY). Using backscatter detection, areas that appeared bright were localized and analyzed.

RESULTS: Light microscopic analysis of the lung sections revealed brown-black foreign material clustered in the perivascular regions and parenchyma (Fig. 2). EDXA analysis of the foreign material in the lungs revealed particles with different combinations of aluminum, silicon, sulfur, potassium, calcium, and iron. (Fig. 3).

CONCLUSIONS: This study illustrates how EDXA can be used to analyze foreign material in the lung (pneumoconiosis). Analysis of lungs with EDXA has been reported previously [2,3], but is only performed in specific cases, and in laboratories where the microscope and necessary equipment are available. Routinely prepared paraffin embedded surgical or autopsy lung tissue can be analyzed with EDXA by using a section from the block. This also allows for correlation to the $H \& E$ section, and retrospective analysis in cases where foreign material was not initially expected. 


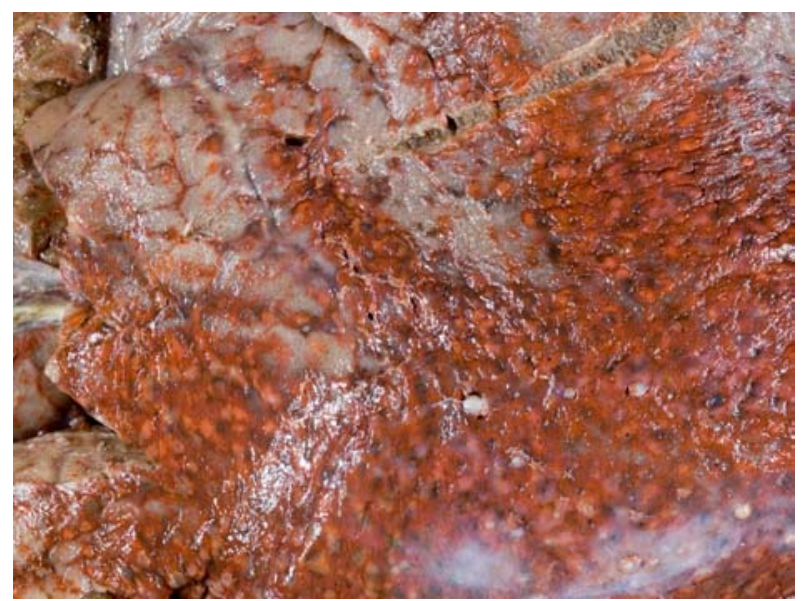

Fig. 1 Gross photo of the lung showing red discoloration.

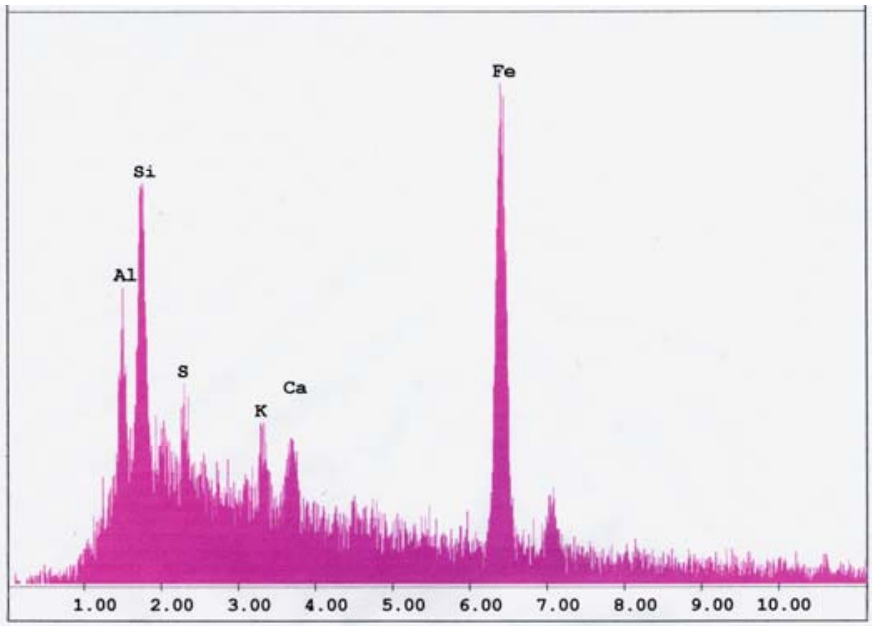

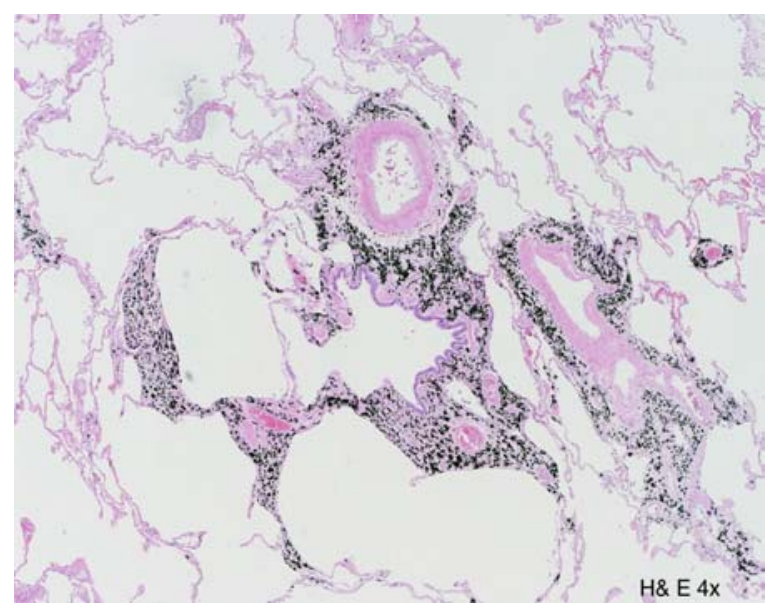

Fig. 2 Light micrograph showing foreign material in the lung (H\&E stain 4x).
Fig. 3 Energy dispersive x-ray spectrum of a particle shows peaks for aluminum, silicon, sulfur, potassium, calcium, and iron.

\section{REFERENCES:}

[1] D. E. Schraufnagel, et al. Inhaled non-infectious toxicants and their effects on the lung. In: Pulmonary Biology in Health and Disease, pg. 395-414. Edited by Bittar EE. $1^{\text {st }}$ ed., Springer, 2002. [2] S. Humble, et al. Titanium particles identified by energy-dispersive x-ray microanalysis within the lungs of a painter at autopsy. Ultrastruct Pathol 27:127-129, 2003.

[3] V. L. Roggli. Scanning electron microscope analysis of mineral fibers in human lungs. In: Microprobe Analysis in Medicine. P. Ingram, J.D. Shelburne, and V.L. Roggli; pg. 97-129. Hemisphere Publishing Corp (Taylor \& Francis Group), 1989. 